\title{
Análisis confirmatorio del instrumento Afrontamiento al Estrés (COPE) en mujeres con violencia en relación de pareja
}

\author{
Confirmatory analysis of the instrument Coping with Stress (COPE) in \\ women with violence in relationship
}

\author{
Gretty A. Canelo Espinoza ${ }^{1}$ \\ Ministerio de la Mujer
}

Recibido: $12-04-18$

\author{
Antonio Serpa Barrientos ${ }^{2}$ \\ Universidad César Vallejo
}

Aceptado: $23-07-18$

\begin{abstract}
Resumen
El uso de los instrumentos psicológicos en el trabajo clínico, en este caso en mujeres con violencia en la relación de pareja, nos pone en constante verificación de la validez de constructo de posibles instrumentos que ayuden a conocer las estrategias de afrontamiento en estos casos. El objetivo de este estudio fue evaluar un aspecto de la validez de constructo, a través de técnicas multivariantes, del Cuestionario de Afrontamiento al Estrés en 267 mujeres, cuyas edades oscilaron entre 18 a 70 años de edad. Se utilizó el cuestionario COPE (versión completa), que consta de 52 ítems caracterizados en tres estilos de afrontamiento con respuesta dicotómica. Se confirmó mediante el uso del Análisis Factorial Confirmatorio y los ajustes del modelo global $(\mathrm{Cmin}=1.83)$, comparativo $(\mathrm{CFI}=.93, \mathrm{GFI}=.91$ y TLI $=$ $.91)$ y otros índices $(\mathrm{RMR}=0.04$ y $\mathrm{RMSEA}=.06)$ arrojaron resultados aceptables y buenos. Así, se concluye que el cuestionario COPE en su versión completa constituye un instrumento válido para la medición de las estrategias de afrontamiento al estrés en mujeres con violencia en la relación de pareja.
\end{abstract}

Palabras clave: Afrontamiento al Estrés; Análisis factorial confirmatorio; validez de constructo y violencia.

\begin{abstract}
The use of psychological instruments in clinical work, in this case in women with violence in the couple relationship, puts us in constant verification of the construct validity of possible instruments that help to know the coping strategies in these cases. The objective of this study was to evaluate an aspect of construct validity, through multivariate techniques, of the Stress Coping Questionnaire in 267 women, whose ages ranged from 18 to 70 years of age. The COPE questionnaire (full version) was used, which consists of 52 items characterized in three coping styles with a dichotomous response. It was confirmed by the use of the Confirmatory Factor Analysis and the adjustments of the global model $(\mathrm{Cmin}=1.83)$, comparative $(\mathrm{CFI}=.93, \mathrm{GFI}=.91$ and $\mathrm{TLI}=.91)$ and other indices $(\mathrm{RMR}=0.04$ and $\mathrm{RMSEA}=.06)$
\end{abstract}

1 Psicóloga Clínica Cem Ministerio de la Mujer Comisaria Chincha.

Autor para correspondencia. Email: gretty.canelo@gmal.com.

2 Docente Tiempo completo Universidad César Vallejo. Email: aserpa@ucv.edu.pe

(C) Los autores. Este artículo es publicado por la Revista de Investigación en Psicología de la Facultad de Psicología, Universidad Nacional Mayor de San Marcos. Este es un artículo de acceso abierto, distribuido bajo los términos de la licencia Creative Commons Atribucion - No Comercia_Compartir Igual 4.0 Internacional. (http://creativecommons.org/licenses/by-nc-sa/4.0/) que permite el uso no comercial, distribución y reproducción en cualquier medio, siempre que la obra original sea debidamente citada. 
yielded acceptable and good results. Thus, it is concluded that the COPE questionnaire in its full version is a valid instrument for the measurement of coping strategies for stress in women with relationship violence.

Keywords: Coping with stress; confirmatory factor analysis; construct validity and violence.

El estrés como concepto deviene de la ingeniería y la física del S. XVII referidos a los materiales que no sufrían deformación sin llegar a la ruptura o fragmentación (Cooper \& Dewe, 2004). Este concepto fue adoptado desde el campo de la biología y medicina a partir de 1920, para referirse a la autorregulación fisiológica u homeostasis de los organismos (Cannon, 1915), lo que sirvió para el desarrollo del concepto de "Síndrome de adaptación general" que explicaba la naturaleza del estrés en los organismos vivos.

Así, la condición de estrés orientada a la salud de las personas se define como el desbalance que ocurre cuando las exigencias internas/externas sobrepasan a los recursos individuales y el organismo fracasa en su adaptación (Selye, 1983), ubicando tres etapas las cuales se dan por la acción directa Estímulo-Respuesta: Fase de alarma, Fase de resistencia y Fase de agotamiento.

Actualmente se reconoce que el estrés es un proceso dinámico donde interactúan factores internos y externos al individuo, quien se ve en la obligación de elaborar una estrategia de afrontamiento (Butler, 1993; Lazarus \& Folkman, 1984). El afrontamiento está relacionado a aspectos conductuales y cognitivos que van generando cambios en el desarrollo continuo y de aquellas exigencias especificas internas y/o externas que son objeto de análisis como demandantes del sistema recursivo de los sujetos (Lazarus \& Folkman, 1984).

Para medir la forma y modos de afrontamiento de un individuo ante una situación estresante, se han desarrollado numerosas escalas con distintos usos y contextos (Kato, 2015); una de las más reconocidas y confiables es la Escala de Modos de Afrontamiento del Estrés (COPE) (Carver, Scheier, \& Weintraub, 1989) que evalúa los estilos de afrontamiento mediante un cuestionario de 52 ítems distribuido en tres estilos de afrontamiento del estrés con 13 áreas o dimensiones; estilo de afrontamiento enfocado en el problema que está orientado a la planificación, la postergación del afronte, recepción y búsqueda de apoyo social y el afrontamiento continuo; por su parte, el estilo de afrontamiento enfocado en la emoción está orientado a dimensiones religiosos, búsqueda constante de soporte emocional, percepción reestructurada de las emociones y el proceso de aceptación; por último, los otros estilos de afrontamiento como el rechazo o negación de las situaciones y por consiguiente las conductas inadecuadas y diversos distracciones (Cassaretto Bardales y Perez-Aranibar, 2016).

Si bien la versión en español ya ha sido validada en nuestro país con buenos niveles de confiabilidad y consistencia (Cassaretto Bardales y Perez-Aranibar, 2016; 
Ticona Benavente, Paucar Quispe y Llerena Callata, 2010); éste instrumento ha sido evaluado en estudiantes universitarios de ambos sexos, con pocas probabilidades de tener problemas de salud. Las evidencias registradas por Casareto y Pérez (2016) concluyen que la estructura factorial confirmatorio del instrumento no se ajusta adecuadamente a la estructura teórica, encontrando los índices ( $\mathrm{GFI}=.80$ y $\mathrm{CFI}=.53)$ por debajo de .90 , como estimador limiten inferior de bondad de ajuste de las evidencias empíricas al modelo teórico.

En ese sentido los diversos reportes entre estrés y el estilo de afrontamiento en mujeres con violencia en la relación de pareja, y es muy frecuente que los pacientes encuentren en las terapias una buena manera de lograr estilos de vida saludables.

Por ello, el objetivo de este estudio es conocer la aproximación de la estructura interna de la Escala de Modos de Afrontamiento al Estrés (COPE) en mujeres con violencia en relación de pareja.

\section{MÉTODO}

La investigación está enmarcado y orientado en evidenciar aspectos psicométricos de los instrumentos de medición, por lo tanto es de tipo instrumental (Montero y Leon, 2007).

Se utilizó un muestreo tipo no probabilístico intencional donde se considera la elección del $100 \%$ de la muestra que cumplen las características estipuladas para la investigación en un determinado tiempo (Tamayo, 2004).

\section{Participantes}

La muestra está constituida por 267 en mujeres con edades entre 17 y 70 años de edad y son víctimas de violencia en relación de pareja, atendidos en un centro de establecimientos de ministerio de salud.

\section{Instrumento}

La Escala de Modos de Afrontamiento al Estrés (COPE) ha sido empleada en diferentes estudios en donde se ha obtenido propiedades psicométricas como la validez de contenido (criterio de jueces o expertos) (Ticona Benavente et al., 2010) y la fiabilidad con valores óptimos (Cassaretto Bardales y Perez-Aranibar, 2016); en ese sentido se utilizó el protocolo y los ítems, previa revisión de criterio de cinco jueces que emitieron opinión en la que consideraron que no se evidencian problemas de redacción y comprensión de los ítems para la muestra designada y su posterior aplicación.

\section{Procedimiento}

El recojo de información se ha prolongado durante seis meses aproximadamente; en el cual, se ha brindado toda la información necesaria acerca de la importancia 
de emplear el instrumento COPE en la evaluación de aquellos pacientes/clientes detectados con indicadores de violencia en relación de pareja, y su posterior aceptación de manera voluntaria a dicha evaluación.

\section{Análisis de datos}

En este análisis se decidió incorporar los tres estilos del afrontamiento del estrés y la constatación se ha obtenido, en un primer momento, por separado con la finalidad de verificar el ajuste del modelo por cada uno de los estilos propuestos; y posteriormente, de manera conjunta estableciendo así la consistencia del modelo, bajo un marco teórico.

También, se usó el análisis de modelos de ecuaciones estructurales (Structural Equation Models; SEM por sus siglas en inglés) mediante el método de Análisis Factorial Confirmatorio con ayuda del programa AMOS v24. Estos modelos estadísticos multivariados llevan ventaja en cuanto a que permite incluir en la estimación los errores de medida tanto en las variables independientes como dependientes a comparación de los modelos de regresión, que son más restrictivos (Lohmöller, 1989).

Por otra parte a considerar son los bondades de ajuste del modelo propuesto se evaluó por medio de los siguientes indicadores: $\mathrm{Cmin} / \mathrm{gl}<3$, índice de raíz del residuo cuadrático promedio estandarizado (SRMR < .05), raíz del residuo cuadrático promedio de aproximación (RMSEA $<.05$ ), el índice de bondad de ajuste comparativo (CFI>.90) (Rodríguez, Ariza, Pérez, \& Mora, 2013) y por último, las estimaciones de las cargas factoriales ha sido considerado como criterio superiores o iguales a .30 (Smits, Timmerman, Barelds \& Meijer, 2015).

\section{RESULTADOS}

Los resultados del análisis de la estructura del instrumento, realizado a través del Análisis Factorial Confirmatorio de los estilos de la Escala de afrontamiento al estrés de $C O P E$, responde a la finalidad de poder verificar el modelo constituido por las tres dimensiones que conforman, para lo cual se ha considerado los mismos indicadores de bondad de ajuste del AFC. Se aprecia que el modelo de tres factores que conforman a la variable latente de la Escala COPE se evidencia valores de Cmin=104.478, con 60 grados de libertad alcanza la proporción de 1,825 (Tabla 1 y Grafico 1).

La constatación de las evidencias empíricas a través del índice residual de la raíz cuadrada media que evalúa la proximidad de la matriz de covarianzas teóricas con la matriz observada, muestra estimaciones pequeñas $(\mathrm{SRMR}=.04)$ y por su parte el índice de Ajuste $(\mathrm{GFI}=.94)$ y Ajuste Ponderado $(\mathrm{AGFI}=.91)$ evidencian resultados aceptables. Así mismo, se obtuvo el índice de bondad de ajuste comparativo (CFI) $=.93 \mathrm{y}$ Taker-Lewis $(\mathrm{TLI})=.91 \mathrm{y}$, por ultimo el índice de raíz cuadrada media de aproximación $($ RMSEA $)=.05$. 
Análisis confirmatorio del instrumento Afrontamiento al Estrés (COPE) en mujeres con violencia en relación de pareja

\section{Tabla 1}

Validez de Constructo de modelo del Estilo de Afrontamiento al estrés de COPE a través del Análisis Factorial Confirmatorio

\begin{tabular}{lc}
\hline Datos & Modelo de 3 factores \\
\hline Chi-Cuadrado mínimo & 109.478 \\
G. L. & 60 \\
Chi-cuadrado mínimo/G.L. & 1.8 \\
RMR & .04 \\
GFI & .94 \\
AGFI & .91 \\
CFI & .93 \\
TLI & .91 \\
RMSEA & .06 \\
\hline
\end{tabular}

G.L: Grados de libertad; RMR: Raíz del residuo cuadrático promedio; GFI: Índice de Ajuste; AGFI: Índice de Ajuste Ponderado; CFI: Índice de bondad de ajuste comparativo; TLI: índice de Taker-Lewis; RMSEA: Raíz del residuo cuadrático promedio de aproximación.

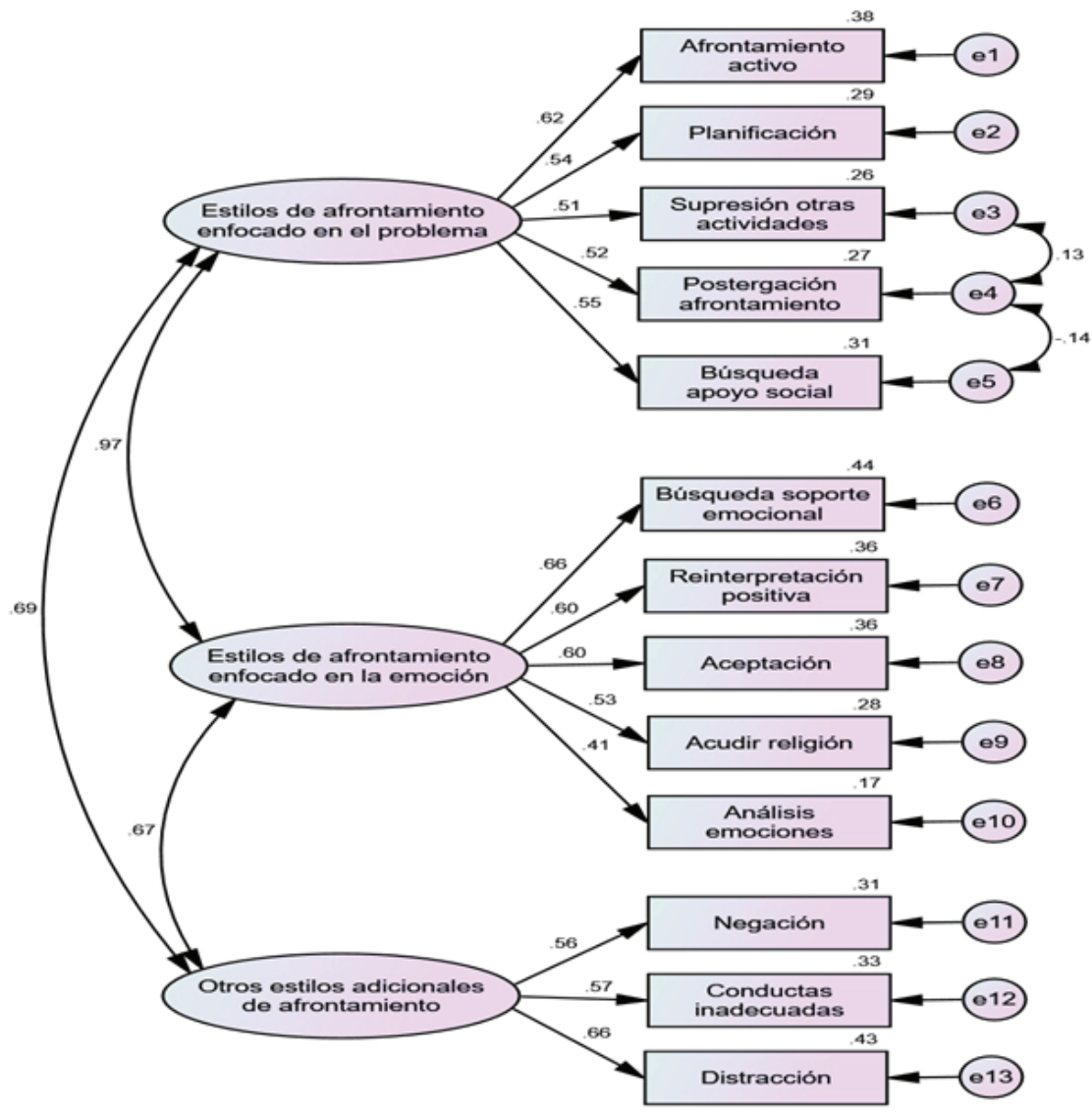

Grafico 1. Modelo de constrcuto de la escala del Estilo de Afrontamiento al estrés de COPE 


\section{DISCUSIÓN}

Los resultados de los índices de ajuste del modelo propuesto por COPE (ver Tabla 1 y Grafico 1) a través del análisis factorial confirmatorio, nos muestran una clara aceptación del modelo propuesto (basado en la teoría) con tres factores.

En consecuencia, se advierte que su aplicación podría sobreestimar la autonomía del paciente o reducir las experiencias de ansiedad (Litman, 2006). Acorde a los resultados obtenidos en nuestro estudio, un estudio anterior (Lyne \& Roger, 2000) sugiere que la evaluación de los otros estilos de afrontamiento, especialmente la negación y la distracción, son fácilmente susceptibles de sesgo de información por parte del encuestado; por ende, si se decide su inclusión en el cuestionario, los resultados deben ser cuidadosamente evaluados.

Es así que, en poblaciones con patologías en donde la ansiedad juega un rol importante (como el alcoholismo), a pesar de que el cuestionario haya obtenido buenos resultados psicométricos, se recomienda su uso con extrema precaución (Hasking \& Oei, 2002). Por ello, considerando que las propiedades psicométricas del COPE fueron buenas, en especial el estilo de afrontamiento orientado a las emociones, registrándose cargas factoriales por encima de la media $(\overline{\mathrm{x}} \geq .30$; Smits, Timmerman, Barelds \& Meijer, 2015) y sin presencia de varianza de error común compartido; por consiguiente, estas estimaciones nos permiten consideran que el modelo estructural es la más adecuada en esta muestra de estudio.

En una de las publicaciones realizadas del instrumento COPE por Casareto y Pérez (2016) evidenciaron índices de ajuste de patrones estructurales no adecuadas $\left(\mathrm{GFI}=.80\right.$ y $\mathrm{CFI}=.53, \mathrm{X}^{2}=1673.26, \mathrm{gl}=87$ y valor $\left.\mathrm{p}<.01\right)$; sin embargo, en esta investigación en mujeres con violencia en la pareja en comparación a una muestra de jóvenes universitarios, los valores de los índices de ajuste registraron un $\mathrm{CFI}=$ $.93, \mathrm{GFI}=.94, \mathrm{X}^{2}=109.478, \mathrm{gl}=60$ y valor $\mathrm{p}<.01$. Estos resultados son concluyentes al confirmar el modelo basado en los tres estilos de afrontamiento al estrés en esta muestra de estudio.

\section{CONCLUSIONES}

La estructura factorial del instrumento de estilos de afrontamiento al estrés de COPE, evidencian un adecuado ajuste de las evidencias empíricas al modelo teórico basado en el estilo de afrontamiento enfocado en el problema, estilo de afrontamiento enfocado en la emoción y otros estilos adicionales al afrontamiento, los valores de ajuste del $\mathrm{X}^{2}=109.478, \mathrm{gl}=60$, valor $\mathrm{p}<.01, \mathrm{CFI}=.93, \mathrm{GFI}=.94$ y RMSEA=.06. Además, para cada estilo de afrontamiento al estrés, las cargas factoriales o pesos factoriales superan la media $(\overline{\mathrm{x}} \geq .30)$ establecida por Smits, Timmerman, Barelds \& Meijer (2015), concluyendo como significativas estos valores obtenidos en esta investigación. 
Y, por último, cabe destacar que este estudio tiene la principal limitación de la selección de la muestra, ya que, además de que fue escogida por conveniencia, pertenece a una población con características específicas: en mujeres con violencia en la relación de pareja, por lo que se recomendaría en futuros estudios considerar como parte del proceso de la validez externa basada en evidencias empíricas.

\section{REFERENCIAS BIBLIOGRÁFICAS}

Butler, G. (1993). Definitions of stress. Occasional paper (Royal College of General Practitioners), (61), 1-5.

Cannon, W. B. (1915). Bodily Changes in Pain, Hunger, Fear, and Rage: An Account of Recent Researches Into the Function of Emotional Excitement. New York: D. Appleton.

Carver, C. S., Scheier, M. F., \& Weintraub, J. K. (1989). Assessing coping strategies: a theoretically based approach. Journal of Personality and Social Psychology, 56(2), 267-283.

Cassaretto Bardales M, \& Perez-Aranibar C. (2016). Afrontamiento al Estrés: Adaptación del Cuestionario COPE en Universitarios de Lima. Revista Iberoamericana de Diagncstico y Evaluacicn Psicologica, 42(2), 95-109.

Cooper, C. L., \& Dewe, P. J. (2004). Stress: A Brief History. New York: Wiley.

Hasking, P. A., \& Oei, T. P. S. (2002). Confirmatory factor analysis of the COPE questionnaire on community drinkers and an alcohol-dependent sample. Journal of Studies on Alcohol, 63(5), 631-640. https://doi.org/10.15288/jsa.2002.63.631

Kato, T. (2015). Frequently Used Coping Scales: A Meta-Analysis. Stress and Health, 31(4), 315-323. https://doi.org/10.1002/smi.2557

Lazarus, R., \& Folkman, S. (1984). Stress, Appraisal, and Coping. New York: Springer Publishing Company.

Litman, J. A. (2006). The COPE inventory: Dimensionality and relationships with approach- and avoidance-motives and positive and negative traits. Personality and Individual Differences, 41(2), 273-284. https://doi.org/10.1016/j.paid.2005.11.032

Lohmöller, J.-B. (1989). Latent Variable Path Modeling with Partial Least Squares. Physica-Verlag.

Lyne, K., \& Roger, D. (2000). A psychometric re-assessment of the COPE questionnaire. Personality and Individual Differences, 29(2), 321-335. https://doi.org/10.1016/ S0191-8869 (99)00196-8

Montero, I., \& León, O. G. (2007). A guide for naming research studies in psychology. International Journal of Clinical and Health Psychology, 7(3), 847-862.

Rodríguez, M. D., Ariza, A. L. G., Pérez, A. H., \& Mora, M. E. D. (2013). Introducción al análisis estadístico multivariado. Barranquilla: Universidad del Norte. 
Selye, H. (1983). The Stress Concept: Past, Present and Future. En Stress Research Issues for the Eighties. (pp. 1-20). New York: John Wiley \& Sons.

Smits, I.A.M., Timmerman, M.E., Barelds, D.P.H., \& Meijer, R.R. (2015). The Dutch symptom checklist-90-revised: is the use of the subscales jusfied?. European Journal of Psychological Assessment, 31(4), 263- 271. doi: 10.1027/1015-5759/a000233

Tamayo, M. T. y. (2004). El proceso de la investigación científica (4ª ed.). México, D.F.: Editorial Limusa.

Ticona Benavente, S. B., Paucar Quispe, G., \& Llerena Callata, G. (2010). Nivel de estrés y estrategias de afrontamiento en estudiantes de la facultad de enfermería: UNSA Arequipa. 2006. Enfermería Global, 1(19), 1-18. 